\title{
Cardiovascular (CV) Risk after Initiation of Abatacept versus TNF Inhibitors in Rheumatoid Arthritis Patients with and without Baseline CV Disease
}

\author{
Yinzhu Jin, Eun Ha Kang, Gregory Brill, Rishi J. Desai, and Seoyoung C. Kim
}

\begin{abstract}
Objective. To evaluate the cardiovascular safety of abatacept (ABA) versus tumor necrosis factor inhibitors (TNFi) in rheumatoid arthritis (RA) patients with and without underlying cardiovascular disease (CVD).

Methods. We identified RA patients with and without baseline CVD who initiated ABA or TNFi by using data from 2 large US insurance claims databases: Medicare (2008-2013) and Truven MarketScan (2006-2015). After stratifying by baseline CVD, ABA initiators were 1:1 propensity score (PS) matched to TNFi initiators to control for $>60$ baseline covariates. Cox proportional hazards regression estimated the HR and 95\% CI for a composite endpoint of CVD including myocardial infarction, stroke/transient ischemic stroke, or coronary revascularization in the PS-matched cohorts. HR from 2 databases were combined through an inverse variance-weighted fixed-effects model.

Results. We included 6102 PS-matched pairs of ABA and TNFi initiators from Medicare and 6934 pairs from MarketScan. Of these, 35.3\% in Medicare and 14.0\% in MarketScan had baseline CVD. $\mathrm{HR}(95 \% \mathrm{CI})$ for composite CVD in the overall ABA group versus TNFi was $0.67(0.55-0.81)$ in Medicare and $1.08(0.83-1.41)$ in MarketScan with the combined HR of 0.79 (0.67-0.92). Among patients with baseline CVD, the HR (95\% CI) was $0.71(0.55-0.92)$ in Medicare and $1.02(0.68-1.51)$ in MarketScan, with the combined HR of 0.79 (0.64-0.98).

Conclusion. In this large cohort of publicly or privately insured patients with RA in the United States, ABA was associated with a $20 \%$ reduced risk of CVD versus TNFi. While this observational study is subject to potential residual confounding, our results were consistent in patients with baseline CVD. (First Release May 15 2018; J Rheumatol 2018;45:1240-8; doi:10.3899/jrheum.170926)
\end{abstract}

Key Indexing Terms:

RHEUMATOID ARTHRITIS BIOLOGIC DISEASE-MODIFYING ANTIRHEUMATIC DRUGS CARDIOVASCULAR DISEASES COMPARATIVE SAFETY RESEARCH

\footnotetext{
From the Division of Pharmacoepidemiology and Pharmacoeconomics, and Division of Rheumatology, Immunology and Allergy, Brigham and Women's Hospital, Harvard Medical School, Boston, Massachusetts, USA; Division of Rheumatology, Department of Internal Medicine, Seoul National University Bundang Hospital, Seongnam, South Korea.

This study was supported by an investigator-sponsored grant from Bristol-Myers Squibb. The study was conducted by the authors independent of the sponsor. The sponsor was given the opportunity to make nonbinding comments on a draft of the manuscript, but the authors retained the right of publication and to determine the final wording. Y. Jin, MS, MPH, Division of Pharmacoepidemiology and Pharmacoeconomics, Brigham and Women's Hospital, Harvard Medical School; E.H. Kang, MD, PhD, MPH, Division of Pharmacoepidemiology and Pharmacoeconomics, Brigham and Women's Hospital, Harvard Medical School, and Division of Rheumatology, Department of Internal Medicine, Seoul National University Bundang Hospital; G. Brill, MS, Division of Pharmacoepidemiology and Pharmacoeconomics, Brigham and Women's Hospital, Harvard Medical School; R.J. Desai, PhD, Division of Pharmacoepidemiology and Pharmacoeconomics, Brigham and Women's Hospital, Harvard Medical School; S.C. Kim, MD, ScD, MSCE, Division of Pharmacoepidemiology and Pharmacoeconomics, and Division of Rheumatology, Immunology and Allergy, Brigham and Women's Hospital, Harvard Medical School.

Address Correspondence to Dr. S.C. Kim, Division of

Pharmacoepidemiology \& Pharmacoeconomics, Brigham and Women's Hospital, 1620 Tremont Street, Suite 3030 Boston, Massachusetts 02120, USA.E-mail: skim62@partners.org

Accepted for publication February 12, 2018.
}

Patients with rheumatoid arthritis (RA) have an increased risk of cardiovascular disease (CVD) compared to the general population $1,2,3,4,5$. Along with traditional CVD risk factors such as hypertension (HTN), dyslipidemia, advanced age, and male sex, markers of RA activity or longterm systemic inflammation also contribute to the increased CVD risk among patients with $\mathrm{RA}^{6,7,8,9}$. A number of studies from the last decade suggest a potential beneficial effect of tumor necrosis factor inhibitors (TNFi) on the risk of CVD in patients with RA compared to nonbiological (nb-) disease-modifying antirheumatic drugs (DMARD) ${ }^{10,11,12}$.

Currently, $5 \mathrm{TNFi}$ and several non-TNFi biologics including abatacept (ABA) are marketed for management of RA in the United States. ABA (CTLA4-Ig) is an effective biologic DMARD that targets $\mathrm{T}$ cell activation and blocks co-stimulation ${ }^{13,14}$. ABA is generally well tolerated and noted to slow the deterioration or decline of $\beta$ cell function and improve glycemic control in some experimental studies ${ }^{15,16}$. However, ABA did not show favorable effects on aortic or arterial stiffness in some small observational studies ${ }^{15,17,18}$. To date, few studies have studied the effect of non-TNFi biologic on cardiovascular (CV) events among patients with

Personal non-commercial use only. The Journal of Rheumatology Copyright $\odot$ 2018. All rights reserved 
RA. In a previous cohort study of older patients with RA (mean age $64 \mathrm{yrs}$ ) who were free of coronary artery disease at the time of biologic initiation, use of TNFi had 30\% elevated risk of acute myocardial infarction (MI) compared to $\mathrm{ABA}^{19}$. While this result is promising for the safety of $\mathrm{ABA}, \mathrm{CV}$ safety of $\mathrm{ABA}$ in other patient populations is unknown.

We therefore sought to examine $\mathrm{CV}$ risks of $\mathrm{ABA}$ versus TNFi in a cohort of patients with RA enrolled in both public and private health plans (i.e., Medicare and MarketScan) in the United States. Since CVD history is one of the strongest risk factors for future $\mathrm{CV}$ events, we assessed the association between ABA and CVD risk specific to baseline CVD status. We hypothesized that ABA use would be associated with a reduced risk of CVD in RA patients with and without baseline CVD.

\section{MATERIALS AND METHODS}

Data source and study cohort. We conducted a cohort study using data from 2 large insurance claims databases: Medicare Parts A/B/D from January 2008 to January 2013, and MarketScan from January 2006 to June 2015. Medicare is a public insurance program for people aged $\geq 65$ years or people with certain disabilities. Part A covers in-hospital stays, Part B generally covers outpatient-based services, and Part D provides prescription drug coverage. The MarketScan database primarily includes the working population, early retirees, and their dependents insured by employer-sponsored plans across the United States. The study protocol was approved by the Institutional Review Board of the Brigham and Women's Hospital, which granted a waiver of informed consent (protocol no. 2015P001708).

We identified patients with RA aged $\geq 18$ older with at least 2 RA International Classification of Diseases, 9th revision (ICD-9) codes (714.xx) separated by $7-365$ days $^{20}$. We required 2 RA ICD- 9 diagnosis codes, both occurring before the index date. Of those, we selected new users of ABA or TNFi (adalimumab, etanercept, certolizumab, golimumab, and infliximab) based on the National Drug Code or J codes. New users were defined as having at least 365 days of continuous insurance enrollment free of the study drugs before the first dispensing or infusion date of ABA or TNFi (i.e., index date). Medicare patients were required to be enrolled in Parts A, B, and D during that time. We excluded patients who received other biologics or tofacitinib in the 365-day pre-index period. Patients were allowed to enter the cohort more than once as long as they met the inclusion and exclusion criteria.

Study subjects were followed from 1 day after the index date until the earliest event of the following: (1) death; (2) outcome occurrence; (3) end of study database period; (4) insurance disenrollment; or (5) last drug available date. Last drug available date was defined as last drug dispensing date plus days of supply of the exposure drug.

Outcome assessment. The primary outcome was a composite CVD endpoint including MI, stroke/transient ischemic attack (TIA) or coronary revascularization. Earliest event date of these 3 events was defined as the outcome occurrence date. MI and stroke/TIA were identified by using inpatient ICD-9 diagnosis code of acute MI (410.x0 and 410.x1) and stroke/TIA (430, 431, 433.x1, 434.x1, 435, 436, and 362.3). Coronary revascularization was identified using ICD-9 procedure codes, current procedural terminology codes, or diagnosis-related group codes. These algorithms have been previously validated and had a positive predictive value $>92 \% \%^{21,22,23}$.

We also included the secondary outcomes that were each a component of composite CVD endpoint, as well as heart failure (HF) and venous thromboembolism (VTE). HF was identified with inpatient ICD-9 diagnosis code (428.x, 398.91, 402.01, 402.11, 402.91, 404.01, 404.11, 404.91, 404.03, 404.13, 404.93), and VTE was identified as inpatient ICD-9 diagnosis codes
(451.1, 451.2, 451.8, 451.9, 453.0-453.4, 453.8, 453.9, 671.3, 671.4, 671.5, 415.1). Patients who had HF or VTE prior to the index date were excluded when we assessed incident HF or VTE outcomes.

Covariates. During the 365-day baseline period prior to the index date, we assessed over 60 variables potentially associated with RA disease severity, biologic initiation, and CVD. These variables were age, sex, calendar year, race (only in Medicare), region (only in Medicare), comorbidities (e.g., smoking status, alcohol use, hyperlipidemia, HTN, atrial fibrillation, HF, coronary heart disease, stroke/TIA, peripheral vascular disease, VTE, chronic liver disease, chronic kidney disease, and diabetes), RA-related medications, other medications, laboratory tests ordered, and healthcare utilization covariates (Table 1A and Table 1B). Baseline CVD was defined as having an inpatient or outpatient diagnosis of coronary heart disease or stroke/TIA prior to the index date.

Statistical analyses. We cross-tabulated baseline characteristics of each cohort (Medicare and MarketScan) by exposure groups (i.e., ABA vs TNFi). After stratifying by the baseline CVD status (CVD+ and CVD- subgroups), we estimated database-specific propensity scores (PS) of starting ABA versus TNFi using multivariable logistic regression models that included all the aforementioned covariates plus index year. Within each database, we then matched ABA initiators to TNFi initiators on PS with a 1:1 ratio using a matching caliper of 0.025 on the PS scale. We assessed covariate balance using standardized differences. The absolute standardized mean difference $<0.1$ was considered as balanced between 2 exposure groups ${ }^{24}$.

In each PS-matched CVD+ and CVD- subgroups and overall cohort, we estimated incidence rate (IR) per 100 person-years (PY) for both primary and secondary outcomes. Separate Cox proportional hazards model estimated HR and $95 \%$ CI for primary and secondary outcomes in each database. PS-matched estimates from the 2 databases were then combined by using an inverse variance weighted fixed-effects model. Proportional hazards assumption was tested by including the interaction term between exposure and followup time and was not violated in any of the models for primary analysis ${ }^{25}$.

Patients could enter the cohort multiple times if they satisfied all the inclusion and exclusion criteria of our study. We accounted for the multiple entry subjects by using sandwich estimator of standard errors in the Cox proportional hazards models ${ }^{26}$. All analyses were conducted in SAS 9.4 (SAS Institute Inc.).

\section{RESULTS}

Cohort characteristics. After applying inclusion and exclusion criteria (Figure 1), we identified 6107 patients with RA who initiated ABA and 25,792 who initiated TNFi in Medicare. In MarketScan, there were 6942 ABA initiators and 65,464 TNFi initiators. Before PS matching, 32.3\% of patients in Medicare and 29.8\% in MarketScan had baseline CVD conditions. As we mention in the Materials and Methods section, we allowed multiple entry to the cohort if a patient met all inclusion/exclusion criteria again. There were $4 \%$ of the patients who entered the cohort more than once.

After 1:1 PS matching, we included a total of 6102 pairs of ABA and TNFi initiators from Medicare and 6934 pairs from MarketScan (Table 1A and Table 1B). Of those, 2156 pairs of ABA initiators and TNFi initiators from Medicare and 968 pairs from MarketScan had baseline CVD (Supplementary Table 1, available from the authors on request). In the main cohort, the mean (SD) age was 73.8 (6.3) and 73.8 (6.5) years for ABA and TNFi initiators in Medicare, respectively, and $56.9(13.0)$ and $56.9(13.4)$ years

Personal non-commercial use only. The Journal of Rheumatology Copyright @ 2018 . All rights reserved. 
Table 1A. Selected baseline demographics and comorbidities after 1:1 PS matching. Values are $\%$ for binary variables and mean \pm SD for continuous variables.

\begin{tabular}{|c|c|c|c|c|}
\hline \multirow[t]{2}{*}{ Characteristics } & \multicolumn{2}{|c|}{ Medicare, $\mathrm{n}=6102$} & \multicolumn{2}{|c|}{ MarketScan, $n=6934$} \\
\hline & $\mathrm{ABA}$ & TNFi & $\mathrm{ABA}$ & TNFi \\
\hline \multicolumn{5}{|l|}{ Demographics } \\
\hline Age & $73.8 \pm 6.3$ & $73.8 \pm 6.5$ & $56.9 \pm 13.0$ & $56.9 \pm 13.4$ \\
\hline Female & 83.1 & 82.8 & 82.1 & 81.2 \\
\hline \multicolumn{5}{|l|}{ Race } \\
\hline White & 85.8 & 85.5 & & \\
\hline Black or African American & 6.6 & 6.6 & & \\
\hline Others & 7.7 & 7.8 & & \\
\hline \multicolumn{5}{|l|}{ Region } \\
\hline Midwest & 20.3 & 20.0 & & \\
\hline Northeast & 13.9 & 14.6 & & \\
\hline South & 44.6 & 44.8 & & \\
\hline West & 18.6 & 18.2 & & \\
\hline \multicolumn{5}{|l|}{ Comorbidities } \\
\hline Hyperlipidemia & 69.3 & 69.9 & 34.2 & 33.1 \\
\hline Hypertension & 80.3 & 81.1 & 44.1 & 44.6 \\
\hline Atrial fibrillation & 14.0 & 13.4 & 4.4 & 4.5 \\
\hline Heart failure & 17.3 & 16.9 & 5.1 & 5.6 \\
\hline Coronary heart disease & 31.6 & 31.8 & 12.2 & 12.1 \\
\hline Stroke/TIA & 7.2 & 7.6 & 2.7 & 2.9 \\
\hline Peripheral vascular disease & 16.2 & 16.3 & 3.8 & 3.7 \\
\hline VTE & 6.9 & 7.0 & 3.3 & 3.9 \\
\hline Chronic liver disease & 9.6 & 9.6 & 5.9 & 5.7 \\
\hline Chronic kidney disease & 14.7 & 15.1 & 4.7 & 4.7 \\
\hline Malignancy & 16.9 & 16.8 & 9.2 & 9.5 \\
\hline COPD & 22.7 & 24.5 & 8.7 & 8.6 \\
\hline Asthma & 14.0 & 14.1 & 10.2 & 9.5 \\
\hline Depression & 20.6 & 20.3 & 13.6 & 12.8 \\
\hline Obesity & 21.6 & 22.9 & 11.2 & 11.3 \\
\hline Smoking & 15.0 & 15.4 & 9.4 & 9.7 \\
\hline \multicolumn{5}{|l|}{ Health care utilization } \\
\hline No. ED visits & $1.0 \pm 3.0$ & $1.0 \pm 2.9$ & $0.7 \pm 1.6$ & $0.7 \pm 1.7$ \\
\hline No. outpatient physician visits & $17.5 \pm 10.0$ & $17.6 \pm 10.4$ & $14.0 \pm 8.8$ & $14.0 \pm 9.6$ \\
\hline Acute hospitalization, $\leq 1 \mathrm{yr}$ & 30.0 & 29.6 & 18.5 & 18.5 \\
\hline \multicolumn{5}{|l|}{ Test ordered } \\
\hline Electrocardiogram & 55.6 & 55.4 & 38.2 & 39.0 \\
\hline Echocardiogram & 30.7 & 30.7 & 18.2 & 18.1 \\
\hline $\mathrm{HbA1C}$ & 25.7 & 26.4 & 18.5 & 18.5 \\
\hline Lipid/cholesterol panel & 49.6 & 50.0 & 38.9 & 38.2 \\
\hline CRP & 58.4 & 59.2 & 55.9 & 56.3 \\
\hline Serum creatinine & 26.7 & 26.9 & 25.6 & 25.4 \\
\hline ESR & 68.4 & 66.5 & 65.0 & 65.3 \\
\hline Blood urea nitrogen & 9.9 & 9.5 & 8.7 & 8.5 \\
\hline Pulmonary function test & 18.5 & 17.7 & 17.2 & 15.7 \\
\hline
\end{tabular}

for ABA and TNFi initiators in MarketScan, respectively. Baseline characteristics were well balanced between the 2 groups with all standardized differences $<0.1$ (Table $1 \mathrm{~A}$ and Table 1B).

In the PS-matched CVD+ subgroup, mean followup times for ABA versus TNFi group were 433.4 (423.0) versus 383.5 (406.6) days in the Medicare cohort, and 401.9 (437.0) versus 424.4 (505.6) days in the MarketScan cohort. Followup times were longer in the CVD- subgroup: the mean followup time for ABA versus TNFi group was 481.5 (449.9) versus 431.9 (426.7) days in the Medicare cohort; 438.6 (507.4) versus
476.4 (542.3) days in the MarketScan cohort. In the MarketScan cohort, mean age was 56 years in the CVD- subgroup, but 65 years in the CVD+ subgroup. Patients with baseline CVD were sicker than those without baseline CVD, with higher prevalence of comorbidities such as diabetes and diabetic complications, HTN, hyperlipidemia, and CVD (Supplementary Table 1, available from the authors on request).

Primary outcome. The overall IR of composite CVD after matching was 2.38 versus 3.58 per $100 \mathrm{PY}$ in the Medicare database for ABA versus TNFi initiators, respectively. 
Table 1B. Selected baseline medications and health care utilization after 1:1 PS matching. Values are \% for binary variables and mean $\pm \mathrm{SD}$ for continuous variables.

\begin{tabular}{|c|c|c|c|c|}
\hline \multirow[t]{2}{*}{ Characteristics } & \multicolumn{2}{|c|}{ Medicare, $\mathrm{n}=6102$} & \multicolumn{2}{|c|}{ MarketScan, $n=6934$} \\
\hline & $\mathrm{ABA}$ & TNFi & $\mathrm{ABA}$ & TNFi \\
\hline \multicolumn{5}{|l|}{ Medications } \\
\hline \multicolumn{5}{|l|}{ Glucocorticoids } \\
\hline Oral glucocorticoids, $\leq 30 \mathrm{~d}$ & 34.6 & 32.9 & 20.5 & 22.0 \\
\hline Oral glucocorticoids, $\leq 1 \mathrm{yr}$ & 73.3 & 71.9 & 50.0 & 53.5 \\
\hline Cumulative dose $^{*}, \leq 1 \mathrm{yr}$ & $1189.1 \pm 1370.1$ & $1166.7 \pm 1470.7$ & $968.5 \pm 3554.7$ & $928.8 \pm 2932.4$ \\
\hline \multicolumn{5}{|l|}{ DMARD } \\
\hline Methotrexate & 54.1 & 61.6 & 36.2 & 47.7 \\
\hline Hydroxychloroquine & 28.5 & 24.0 & 21.5 & 20.4 \\
\hline Leflunomide & 20.0 & 16.3 & 13.8 & 10.9 \\
\hline Other nonbiologics & 19.0 & 16.0 & 12.7 & 12.0 \\
\hline No. unique nonbiologic DMARD & D $\quad 1.2 \pm 0.8$ & $1.2 \pm 0.8$ & $0.8 \pm 0.9$ & $0.9 \pm 0.9$ \\
\hline \multicolumn{5}{|c|}{ Cardiovascular drugs } \\
\hline Beta blockers & 38.5 & 37.9 & 16.8 & 17.1 \\
\hline Calcium channel blockers & 28.6 & 29.3 & 12.4 & 12.6 \\
\hline ACEI/ARB & 49.8 & 50.5 & 23.7 & 23.8 \\
\hline Nitrates & 8.6 & 8.4 & 2.6 & 2.4 \\
\hline Statins & 44.2 & 45.8 & 19.4 & 19.3 \\
\hline Nonstatin lipid lowering drugs & 9.0 & 8.9 & 5.8 & 6.1 \\
\hline Antiplatelets & 11.1 & 11.4 & 3.8 & 4.1 \\
\hline Anticoagulants & 12.7 & 12.5 & 5.5 & 5.7 \\
\hline Anti-arrhythmics & 3.2 & 2.7 & 0.9 & 1.0 \\
\hline Loop diuretics & 24.3 & 24.8 & 9.1 & 9.6 \\
\hline Thiazide diuretics & 29.8 & 30.3 & 16.0 & 15.5 \\
\hline Other diuretics & 9.6 & 10.1 & 5.6 & 5.7 \\
\hline \multicolumn{5}{|l|}{ Analgesics } \\
\hline NSAID & 38.3 & 37.6 & 31.6 & 32.0 \\
\hline COXIB & 10.7 & 10.7 & 8.1 & 7.6 \\
\hline Opioids, $\leq 30 \mathrm{~d}$ & 34.6 & 34.1 & 20.5 & 19.2 \\
\hline \multicolumn{5}{|l|}{ Others } \\
\hline Insulin & 10.0 & 9.7 & 5.3 & 5.6 \\
\hline DPP4 inhibitors & 2.4 & 2.8 & 1.4 & 1.6 \\
\hline Benzodiazepines & 6.4 & 6.7 & 16.8 & 17.3 \\
\hline Bisphosphonates & 23.1 & 22.9 & 9.9 & 10.3 \\
\hline PPI & 46.4 & 46.7 & 26.3 & 26.0 \\
\hline No. unique generics & $14.8 \pm 6.8$ & $14.8 \pm 6.9$ & $9.9 \pm 9.1$ & $9.9 \pm 8.1$ \\
\hline
\end{tabular}

\footnotetext{
* Prednisone equivalent dose in milligrams. ABA: abatacept; ACEI: angiotensin converting enzyme inhibitor; ARB: angiotensin receptor blocker; COPD: chronic obstructive pulmonary disease; COXIB: cyclooxygenase 2 inhibitors; CRP: C-reactive protein; DMARD: disease-modifying antirheumatic drugs; DPP4: dipeptidyl peptidase-4; ED: emergency department; ESR: erythrocyte sedimentation rate; HbA1C: hemoglobin A1C; NSAID: nonsteroidal anti-inflammatory drug; PPI: proton pump inhibitor; PS: propensity score; TIA: transient ischemic attack; VTE: venous thromboembolism; TNFi: tumor necrosis factor inhibitors.
}

Compared to IR in Medicare, MarketScan database IR were lower for both groups (1.38 per $100 \mathrm{PY}$ for $\mathrm{ABA}$ and 1.27 per 100 PY for TNFi initiators; Table 2). Patients with baseline CVD had higher IR than those without.

Overall combined HR for composite CVD associated with ABA initiation versus TNFi from the 2 databases was 0.79 (95\% CI 0.67-0.92). In the Medicare cohort, there was a significant association between ABA and lower CVD risk compared with TNFi initiators (HR 0.67, 95\% CI 0.55-0.81). However, there was no significant difference in the CVD risk between the ABA or TNFi groups among MarketScan cohort (HR 1.08, 95\% CI 0.83-1.41). Combined HR for the risk of composite CVD among the CVD+ subgroup was 0.79 (95\% CI 0.64-0.98). Among the CVD- subgroup, HR for composite CVD associated with $\mathrm{ABA}$ versus TNFi was 0.61 (95\% CI 0.46-0.82) in Medicare and 1.10 (95\% CI $0.77-1.57)$ in MarketScan, with the combined HR of 1.04 (95\% CI 0.74-1.46).

Secondary outcomes. In the overall cohort, the combined HR of coronary revascularization was numerically lower but not statistically significant for ABA initiators compared to TNFi initiators in both the Medicare and MarketScan cohorts (Figure 2). We observed a significant reduction in MI risk among ABA initiators in the Medicare overall cohort (HR

$$
\text { Personal non-commercial use only. The Journal of Rheumatology Copyright (C) 2018. All rights reserved. }
$$




\section{Medicare Cohort}

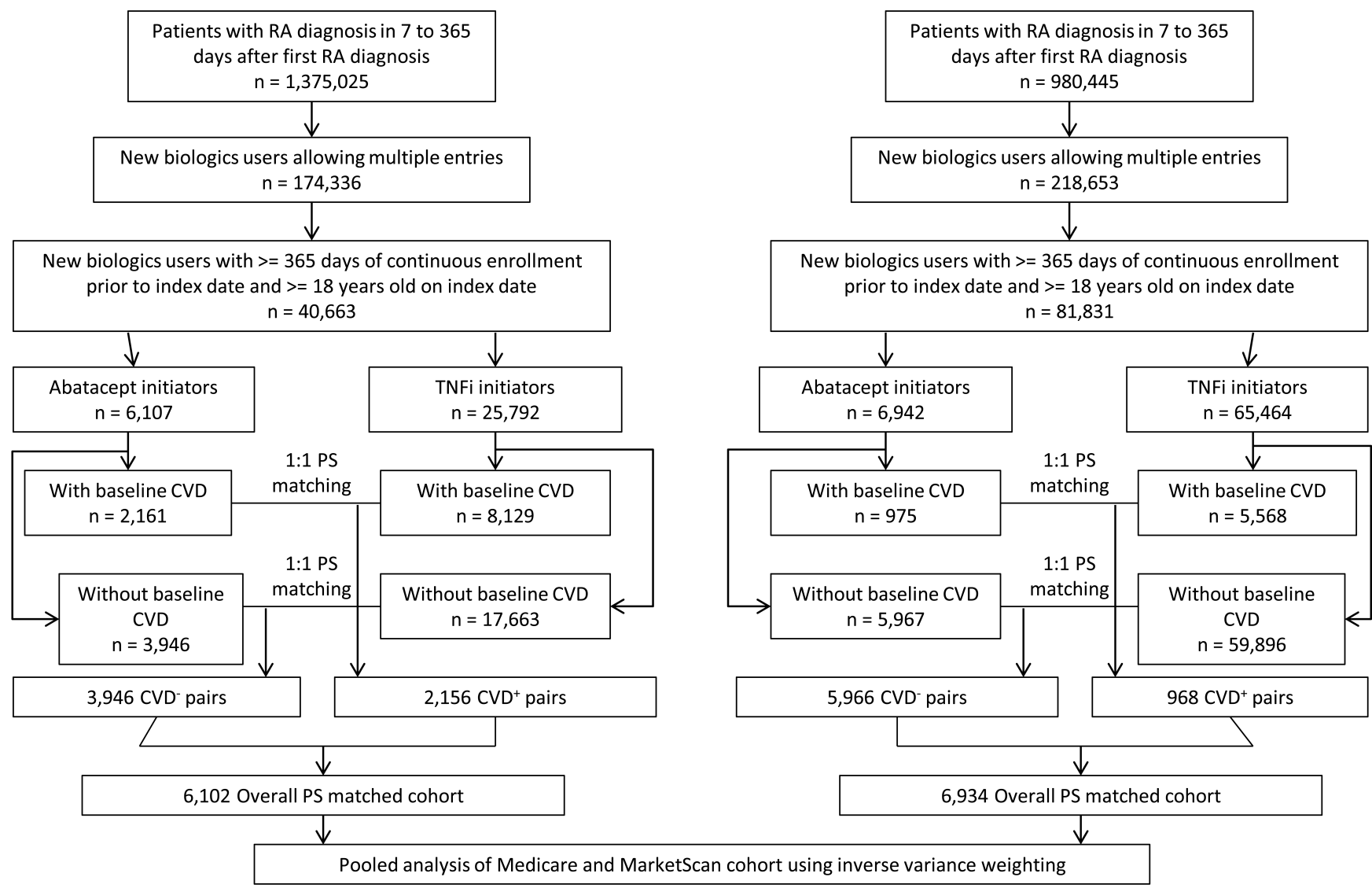

Figure 1. Flow chart of study cohort selection. CVD: cardiovascular disease; PS: propensity score; RA: rheumatoid arthritis; TNFi: tumor necrosis factor inhibitors.

Table 2. Risk of composite CVD outcome* in ABA initiators versus TNFi: 1:1 PS matched analysis.

\begin{tabular}{|c|c|c|c|c|c|c|}
\hline \multicolumn{7}{|l|}{ Overall } \\
\hline Medicare & $\mathrm{ABA}$ & 6102 & 185 & 2.38 & $0.67(0.55-0.81)$ & $0.79(0.67-0.92)$ \\
\hline \multirow[t]{2}{*}{ MarketScan } & $\mathrm{ABA}$ & 6934 & 114 & 1.38 & $1.08(0.83-1.41)$ & \\
\hline & TNFi & 6934 & 113 & 1.27 & Ref & \\
\hline \multicolumn{7}{|c|}{ With baseline CVD } \\
\hline \multirow[t]{2}{*}{ MarketScan } & $\mathrm{ABA}$ & 968 & 49 & 4.60 & $1.02(0.68-1.51)$ & \\
\hline & TNFi & 968 & 49 & 4.35 & Ref & \\
\hline \multicolumn{7}{|c|}{ Without baseline CVD } \\
\hline \multirow[t]{2}{*}{ Medicare } & $\mathrm{ABA}$ & 3946 & 78 & 1.50 & $0.61(0.46-0.82)$ & $1.04(0.74-1.46)$ \\
\hline & TNFi & 3946 & 114 & 2.44 & Ref & \\
\hline MarketScan & $\mathrm{ABA}$ & 5966 & 65 & 0.91 & $1.10(0.77-1.57)$ & \\
\hline
\end{tabular}

${ }^{*}$ Composite CVD is any of MI, stroke/TIA or coronary revascularization. CVD: cardiovascular disease; ABA: abatacept; TNFi: tumor necrosis factor inhibitors; PS: propensity score; IR: incidence rate; MI: myocardial infarction; PY: person-years; TIA: transient ischemic attack.

$0.56,95 \%$ CI $0.41-0.76)$ compared to TNFi initiators; after combining the 2 databases, the HR was 0.71 (95\% CI $0.55-0.93)$. There was no significant association between
ABA and other secondary outcomes (incident HF, incident VTE, stroke/TIA) in the overall cohort.

In the CVD+ subgroup, we observed a significant associ-

\section{Personal non-commercial use only. The Journal of Rheumatology Copyright @ 2018 . All rights reserved.}






Figure 2. Risk of secondary outcomes for ABA versus TNFi use after PS matching in overall cohort. ABA: abatacept; HF: heart failure; MI: myocardial infarction; PS: propensity score; TIA: transient ischemic stroke; TNFi: tumor necrosis factor inhibitors; VTE: venous thromboembolism.

ation between $\mathrm{ABA}$ and a reduced risk of coronary revascularization by $65 \%$ in MarketScan (HR $0.35,95 \% \mathrm{CI}$ 0.16-0.77; Figure 3); after combining the 2 databases, the combined HR was 0.63 (95\% CI 0.44-0.92). The risk of MI was also reduced in $\mathrm{ABA}$ initiators, similar to what we observed in the overall cohort (HR 0.58, 95\% CI 0.39-0.87 in the Medicare cohort; combined HR 0.64, 95\% CI 0.44-0.92). No significant association was observed between ABA use and other secondary outcomes (incident HF, incident VTE, stroke/TIA) in the CVD+ subgroup.

In the CVD- subgroup, combined HR of any of the secondary results were not significantly different between ABA and TNFi. However, in the Medicare cohort, we consistently found an association between ABA use and a lower risk of MI (HR 0.52, 95\% CI 0.32-0.83; Figure 4), as well as stroke/TIA (HR 0.61, 95\% CI 0.41-0.92).

\section{DISCUSSION}

In this large observational study including Medicare-enrolled elderly patients and commercially insured younger patients, we found that the risk of a composite CVD endpoint, including MI, stroke, and revascularization, was lower in patients with RA who initiated ABA compared with those who initiated a TNFi. Further, such association was consistently noted among high risk groups such as the older population (Medicare cohort) and patients with baseline CVD.
From the primary outcome analyses in overall cohort and subgroups, we observed that IR was highest in TNFi initiators in the Medicare CVD+ subgroup (5.92 per $100 \mathrm{PY}$ ). Combined estimates demonstrated ABA initiators had $20 \%$ reduction in composite CVD risk versus TNFi initiators in overall and CVD+ subgroup. However, there was no significant difference in the composite CVD risk among patients without baseline CVD. In the Medicare cohort, we observed a more pronounced relative risk in the CVD- subgroup than CVD+ subgroup. It is possibly because of the larger difference in IR of TNFi initiators in the 2 subgroups. The absolute IR difference between ABA and TNFi initiators was still greater in the CVD+ subgroup (-1.74 per $100 \mathrm{PY})$ compared to that in CVD- subgroup (-0.94 per $100 \mathrm{PY})$. To our knowledge, no previous studies have shown such a difference in CVD risk associated with use of ABA between RA patients with and without underlying CVD conditions.

Unlike the results from Medicare, in MarketScan we found no associations between ABA and CVD risk in the overall group or any of the CVD subgroups. Different results from the 2 databases might be partially explained by the difference in mean age since Medicare primarily includes patients aged $\geq 65$ years. Because of the relatively smaller number of patients aged $\geq 65$ years in MarketScan, however, we could not fully investigate the potential effect modification by age on the CV effect of ABA. Further, MarketScan Personal non-commercial use only. The Journal of Rheumatology Copyright @ 2018 . All rights reserved. 


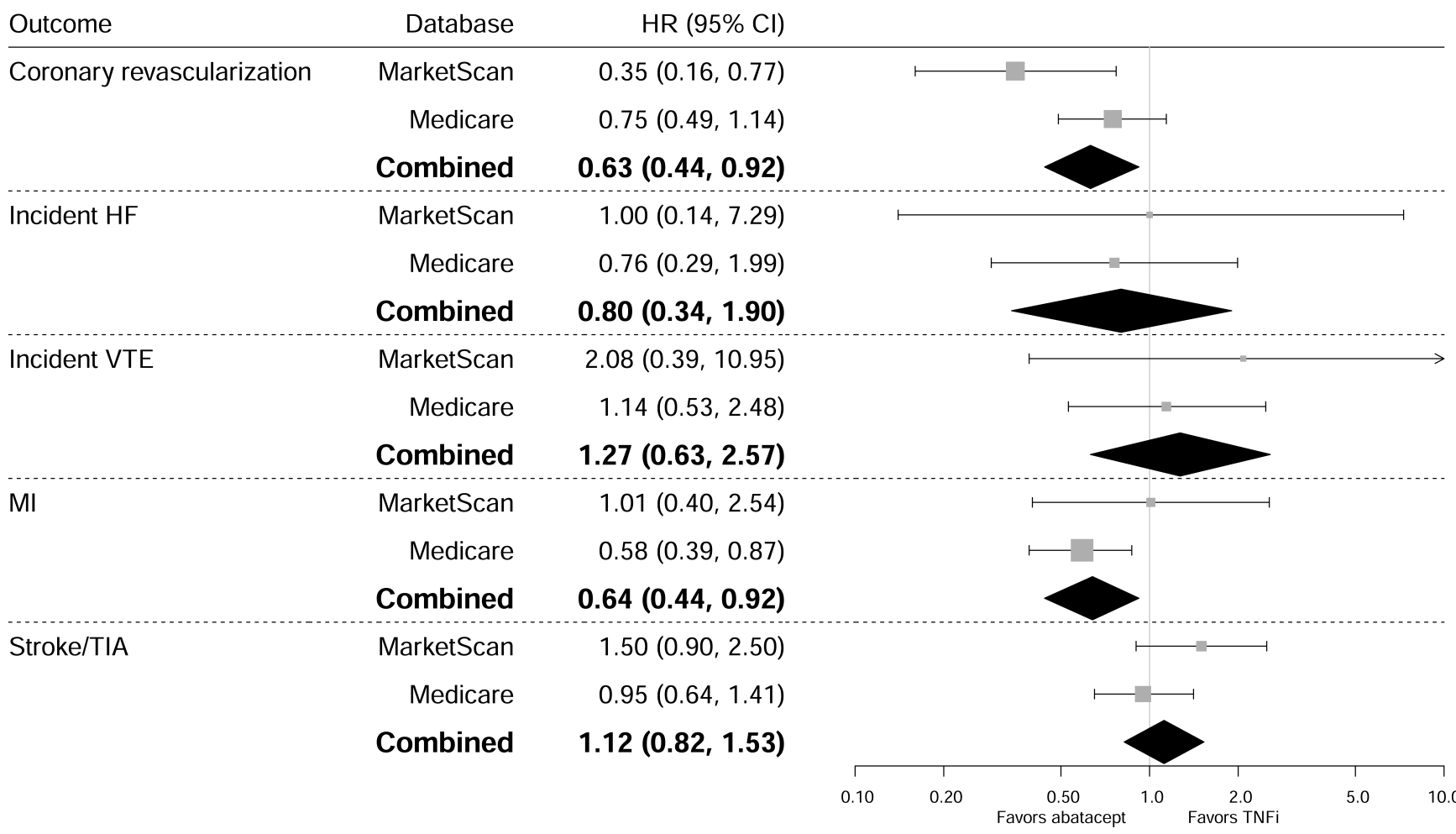

Figure 3. Risk of secondary outcomes for ABA versus TNFi after PS matching in baseline CVD+ subgroup. ABA: abatacept; CVD: cardiovascular disease; HF: heart failure; MI: myocardial infarction; PS: propensity score; TIA: transient ischemic stroke; TNFi: tumor necrosis factor inhibitors; VTE: venous thromboembolism.

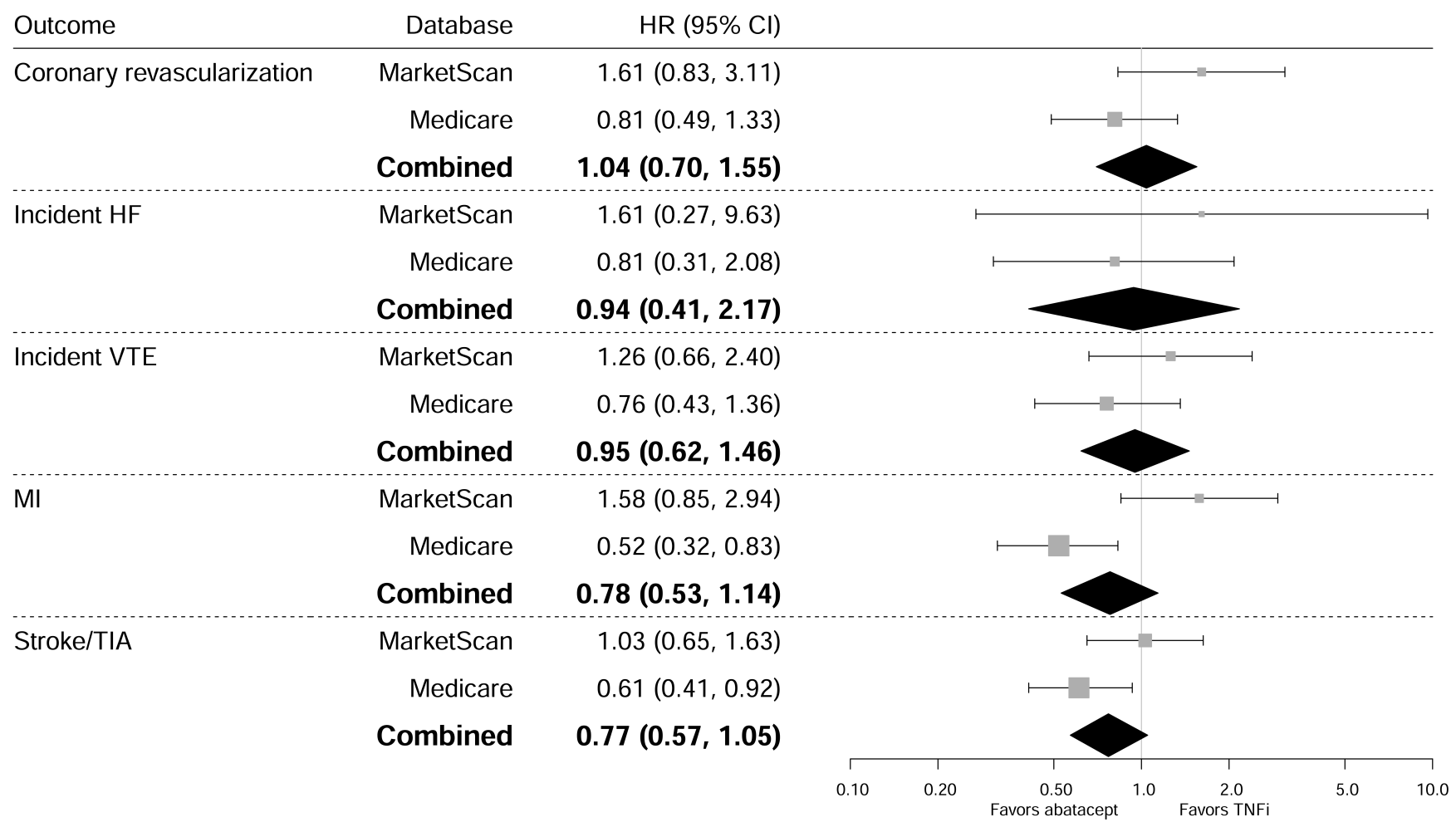

Figure 4. Risk of secondary outcomes for ABA versus TNFi after PS matching in baseline CVD- subgroup. ABA: abatacept; CVD: cardiovascular disease; HF: heart failure; MI: myocardial infarction; PS: propensity score; TIA: transient ischemic stroke; TNFi: tumor necrosis factor inhibitors; VTE: venous thromboembolism. 
tends to have healthier enrollees than Medicare given the same age groups. Difference in the socioeconomic status and physical activity level in the 2 databases may also partly play a role in the results. However, we did not have information on these variables.

We also examined each component of primary outcomes (MI, coronary revascularization, and stroke/TIA) plus 2 other common CVD (incident HF and incident VTE) as secondary outcomes. Overall, we observed a protective trend of ABA compared to TNFi after combining estimates from the 2 databases (Figure 2, Figure 3, and Figure 4), although most of the associations were not significant; this is possibly related to the smaller sample size and number of outcomes, which resulted in reduced power.

Our results for the secondary outcome of MI are similar to the findings from Zhang, et $a l^{19}$. The authors conducted a retrospective cohort in Medicare enrollees from 2006 to 2012 and demonstrated that TNFi initiators had a $30 \%$ higher risk of acute MI compared with ABA initiators. In the Medicare cohort, we observed a $44 \%$ reduction in the risk of $\mathrm{MI}$ in ABA initiators compared to TNFi initiators. Subgroup analyses also demonstrated similar results. After combining results from the 2 databases, the risk reduction in $\mathrm{ABA}$ versus TNFi was $36 \%$ in the CVD+ subgroup and $22 \%$ in the CVDsubgroup.

Although we observed a trend of potentially protective effect of ABA in Medicare, there was an increased risk of incident VTE in ABA users compared to TNFi in the baseline CVD+ subgroup from both Medicare and MarketScan. There is limited evidence about ABA safety profile regarding VTE outcome, and our study has limited power to further explain the observed result. Future studies are needed to examine the effect of ABA on VTE.

Our study has several strengths. First, we examined 2 large nationwide databases with different baseline characteristics. Previous studies mostly focused on older patients with RA and we could not fully understand the comparative CV safety of TNFi or non-TNF biologics among RA patients with different $\mathrm{CV}$ risk profiles. Here we demonstrated that ABA has more favorable $\mathrm{CV}$ safety among patients with RA at high $\mathrm{CV}$ risk (i.e., those with baseline CVD conditions or advanced age). Second, our study population is representative of both publicly and commercially insured populations in the United States. Third, by stratifying the cohort into with and without baseline CVD subgroups, we were able to provide more specific evidence for physicians regarding treatment decisions for patients with different baseline risks. Finally, we conducted a comprehensive assessment of 65 covariates including healthcare utilization and physicians' orders for laboratory tests, and we used 1:1 PS matching to better adjust for the baseline confounding between the 2 exposure groups.

Our study has several limitations. First, as inherent in any observational study, our study is subject to confounding by partially measured or unmeasured covariates. TNFi initiators and ABA initiators might have different disease severity and activity, but such data were not available in the study databases. However, to better account for unmeasured balance RA disease activity and severity in our study cohorts, we included baseline covariates such as use of steroids, nonsteroidal antiinflammatory drugs, opioids, and other DMARD, as well as visits to rheumatologists and other healthcare utilization patterns and physicians' orders for laboratory tests in the PS model; these variables can provide indirect information related to RA disease severity. Further, using the new user design with an active comparator (i.e., the TNFi group), we tried to reduce the confounding by indication $^{27}$. In the PS-matched cohort from each database, use of nbDMARD, oral glucocorticoids in 30 or 365 days, and the cumulative dose of oral glucocorticoids prior to the index date were well balanced. Nonetheless, residual confounding remains a potential issue in our study. Second, we conducted 1:1 PS matching, which has limited the number of patients in each group, thus leading to potentially inadequate statistical power for some secondary analyses. Third, potential misclassification of comorbidities and outcomes is possible as we mainly relied on billing diagnosis and procedure codes. Because smoking or excess alcohol use can increase a risk of CVD, we used claims-based algorithms to identify patients who smoked ${ }^{28}$ or consumed alcohol at baseline. However, it is likely that these variables were underrecorded because our algorithms mostly recorded patients who had severe use of tobacco or alcohol. Fourth, we required all patients to be actively enrolled at least 1 year prior to the index date for covariate assessment. However, this period may not have been adequately long to determine patients' baseline CV risk or RA severity or duration. Because we used insurance claims databases, we needed to rely on patients' active enrollment status in a given health plan.

Our present study, based on 2 large RA cohorts enrolled in commercial health plans or Medicare in the United States, suggests a more favorable $\mathrm{CV}$ safety of ABA compared to TNFi, particularly among RA patients with advanced age and presence of CVD at baseline. The results provide important population-based head-to-head comparison data that could guide physicians' treatment decision for patients with RA in clinical practice.

\section{REFERENCES}

1. Gabriel SE. Cardiovascular Morbidity and mortality in rheumatoid arthritis. Am J Med 2008;10 Suppl 1:S9-14.

2. Solomon DH, Karlson EW, Rimm EB, Cannuscio CC, Mandl LA, Manson JE, et al. Cardiovascular morbidity and mortality in women diagnosed with rheumatoid arthritis. Circulation 2003;107:1303-7.

3. Maradit-Kremers H, Crowson CS, Nicola PJ, Ballman KV, Roger VL, Jacobsen SJ, et al. Increased unrecognized coronary heart disease and sudden deaths in rheumatoid arthritis: A population-based cohort study. Arthritis Rheum 2005;52:402-11.

4. DeMaria AN. Relative risk of cardiovascular events in patients with rheumatoid arthritis. Am J Cardiol 2002;89:33D-8D.

5. Avina-Zubieta JA, Thomas J, Sadatsafavi M, Lehman AJ, Lacaille

Personal non-commercial use only. The Journal of Rheumatology Copyright (C) 2018. All rights reserved. 
D. Risk of incident cardiovascular events in patients with rheumatoid arthritis: a meta-analysis of observational studies. Ann Rheum Dis 2012;71:1524-9.

6. Solomon DH, Avorn J, Katz JN, Weinblatt ME, Setoguchi S, Levin $\mathrm{R}$, et al. Immunosuppressive medications and hospitalization for cardiovascular events in patients with rheumatoid arthritis. Arthritis Rheum 2006;54:3790-8.

7. Dessein PH, Joffe BI, Veller MG, Stevens BA, Tobias M, Reddi K, et al. Traditional and nontraditional cardiovascular risk factors are associated with atherosclerosis in rheumatoid arthritis. J Rheumatol 2005;32:435-42.

8. Solomon DH, Kremer J, Curtis JR, Hochberg MC, Reed G, Tsao P, et al. Explaining the cardiovascular risk associated with rheumatoid arthritis: traditional risk factors versus markers of rheumatoid arthritis severity. Annals Rheum Dis 2010;69:1920-5.

9. Liao KP, Solomon DH. Traditional cardiovascular risk factors, inflammation and cardiovascular risk in rheumatoid arthritis. Rheumatology 2013;52:45-52.

10. Roubille C, Richer V, Starnino T, McCourt C, McFarlane A, Fleming $\mathrm{P}$, et al. The effects of tumour necrosis factor inhibitors, methotrexate, non-steroidal anti-inflammatory drugs and corticosteroids on cardiovascular events in rheumatoid arthritis, psoriasis and psoriatic arthritis: a systematic review and meta-analysis. Ann Rheum Dis 2015;74:480-9.

11. Avouac J, Allanore Y. Cardiovascular risk in rheumatoid arthritis: effects of anti-TNF drugs. Expert Opin Pharmacother 2008; 9:1121-8.

12. Greenberg JD, Kremer JM, Curtis JR, Hochberg MC, Reed G, Tsao $\mathrm{P}$, et al. Tumour necrosis factor antagonist use and associated risk reduction of cardiovascular events among patients with rheumatoid arthritis. Ann Rheum Dis 2011;70:576-82.

13. Genovese MC, Becker J-C, Schiff M, Luggen M, Sherrer Y, Kremer $\mathrm{J}$, et al. Abatacept for rheumatoid arthritis refractory to tumor necrosis factor $\alpha$ inhibition. N Engl J Med 2005;353:1114-23.

14. Kremer JM, Westhovens R, Leon M, Di Giorgio E, Alten R, Steinfeld $\mathrm{S}$, et al. Treatment of rheumatoid arthritis by selective inhibition of T-cell activation with fusion protein CTLA4Ig. N Engl J Med 2003;349:1907-15.

15. Ursini F, Russo E, Letizia Hribal M, Mauro D, Savarino F, Bruno C, et al. Abatacept improves whole-body insulin sensitivity in rheumatoid arthritis: an observational study. Medicine 2015;94:e888.

16. Orban T, Bundy B, Becker DJ, DiMeglio LA, Gitelman SE, Goland $\mathrm{R}$, et al; Type 1 Diabetes TrialNet Abatacept Study Group. Costimulation modulation with abatacept in patients with recent-onset type 1 diabetes: follow-up 1 year after cessation of treatment. Diabetes Care 2014;37:1069-75.
17. Ursini F, Mauro D, Naty S, Gagliardi D, Grembiale RD. Improvement in insulin resistance after short-term treatment with abatacept: case report and short review. Clin Rheumatol 2012;31:1401-2.

18. Mathieu S, Couderc M, Glace B, Pereira B, Tournadre A, Dubost $\mathrm{J}-\mathrm{J}$, et al. Effects of 6 months of abatacept treatment on aortic stiffness in patients with rheumatoid arthritis. Biologics 2013; 7:259-64.

19. Zhang J, Xie F, Yun H, Chen L, Muntner P, Levitan EB, et al. Comparative effects of biologics on cardiovascular risk among older patients with rheumatoid arthritis. Ann Rheum Dis 2016;75:1813-8.

20. Kim SY, Servi A, Polinski JM, Mogun H, Weinblatt ME, Katz JN, et al. Validation of rheumatoid arthritis diagnoses in health care utilization data. Arthritis Res Ther 2011;13:R32.

21. Kiyota Y, Schneeweiss S, Glynn RJ, Cannuscio CC, Avorn J, Solomon DH. Accuracy of Medicare claims-based diagnosis of acute myocardial infarction: estimating positive predictive value on the basis of review of hospital records. Am Heart J 2004;148:99-104.

22. Andrade SE, Harrold LR, Tjia J, Cutrona SL, Saczynski JS, Dodd $\mathrm{KS}$, et al. a systematic review of validated methods for identifying cerebrovascular accident or transient ischemic attack using administrative data. Pharmacoepidemiol Drug Saf 2012;21 Suppl 1:100-28.

23. Kumamaru H, Judd SE, Curtis JR, Ramachandran R, Hardy NC, Rhodes JD, et al. Validity of Claims-Based Stroke Algorithms in Contemporary Medicare Data: REGARDS Study Linked with Medicare Claims. Circ Cardiovasc Qual Outcomes 2014;7:611-9.

24. Austin PC. Using the standardized difference to compare the prevalence of a binary variable between two groups in observational research. Commun Stat Simul Comput 2009;38:1228-34.

25. Kleinbaum DG, Klein M. Evaluating the proportional hazards assumption. In: Survival analysis: a self-learning text, third edition. New York: Springer New York; 2012:161-200.

26. Lee EW, Wei LJ, Amato DA, Leurgans S. Cox-type regression analysis for large numbers of small groups of correlated failure time observations. In: Klein JP, Goel PK, editors. Survival analysis: state of the art. Dordrecht: Springer Netherlands; 1992:237-47.

27. Yoshida K, Solomon DH, Kim SC. Active-comparator design and new-user design in observational studies. Nature Rev Rheumatol 2015;11:437-41.

28. Desai RJ, Solomon DH, Shadick N, Iannaccone C, Kim SC. Identification of smoking using Medicare data - a validation study of claims-based algorithms. Pharmacoepidemiol Drug Saf $2016 ; 25: 472-5$. 